\title{
stabilisation et consolidation de la colline d'Issy-les-Moulineaux
}

\author{
par \\ C. Dufour \\ Géotechnique Appliquée, Boulogne
}

RESUME - La colline d'Issy les Moulineaux a été exploitée en carrières souterraines pour obtenir de la craie et du calcaire au 19 ème siècle. De nombreux effondrements se sont produits à une époque où cette région était encore une campagne, mais l'urbanisation du site sans précautions a amené une catastrophe en 1961. Il s'agissait donc de travailler à la reconstruction et à la consolidation du site. Grace à l'aide des pouvoirs publics, en particulier de l'Inspection Générale des Carrières, \& de la municipalité, un groupe de techniciens a pu proposer un projet de consolidation à l'échelle du problème. La zone actuellement consolidée concerne environ 15 hectares, l'ensemble du projet de consolidation sera terminé en 1985. Les travaux sont réalisés de manière très classique, par contre la conception de ces travaux est faite en réunissant la mécanique des sols et la statique générale et en évitant tout pointillisme de détail.

\section{1) GENERALITES :}

A la suite d'un effondrement de deux hectares en 1961, sous l'égide de Mr Gastinne propriétaire d'un tir aux pigeons sur la colline d'Issy les Mx, en collaboration avec I'Inspection Générale des Carrières, les services techniques de la ville d'Issy les Mx, le bureau Véritas et l'Atelier Gambert, notre société Géotechnique Appliquée a été chargée des études de sols en vue de permettre une stabilisation définitive des terrains privés et municipaux se situant sur la colline. Cette opération au départ de caractère semi-public a abouti à la création d'une zone d'aménagement concerté à l'échelle des projets de consolidation s'élevant, non compris le prix des terrains, à la somme de 26000000 Frs, valeur Janvier 1971. La charge foncière a été calculée à partir de cette estimation et du prix des terrains et un promoteur privé, le groupe Drouot représenté par la SEERI, a pris en charge 1'opération d'urbanisme comprenant des logements (1700), des commerces, des groupes scolaires, foyer de personnes agées, crèche, gymnase, parc municipal et tennis; la surface des planchers hors sous-sols et équipements étant de $177000 \mathrm{~m} 2$ pour un terrain de 83390 mètres carrés.

La zone sous minée comprend $250000 \mathrm{~m} 3$ de vides visitables \& nécessitera $350000 \mathrm{~m} 3$ d'injection de sablon ciment correspondant au remplissage de $13 \mathrm{~km}$ de galeries variant de 4 à $10 \mathrm{~m}$ de hauteur et de 4 à $6 \mathrm{~m}$ de largeur La fondations des bâtiments dans les effondrements nécessitera l'exécution de 160 puits de $1.80 \mathrm{~m}$ de diamètre et de $55 \mathrm{~m}$ de profondeur. L'objet de la présente communication est de montrer d'une part comment on en est arrivé au stade de l'instabilité, et d'autre part de préciser les hypothèses de consolidation retenues.

Enfin il est important de noter que, exceptionnellement, il s'agit d'un projet uniquement de techniciens très activement soutenu par les pouvoirs publics ; en effet, l'intervention de la puissance financière n'a eu lieu qu'après l'accord de réalisation de ZAC et l'établissement complet du projet de reprise.

\section{2) HISTORIQUE :}

La carte de la fig 1 , bien qu'en noir et blanc, montre l'enchevêtrement des galeries sous la colline, les zones les plus sombres correspondent à deux étages de carrières de craie, les zones irrégulières à un étage supplémentaire d'exploitation de calcaire grossier.

La coupe de la fig 2 montre le contexte géologique de la colline avec en partie haute les carrières de calcaire grossier souterraines ou à ciel ouvert, en partie basse les carrières de craie sur un étage de $8 \mathrm{~m}$ à la partie droite ou deux étages de 4 et $6 \mathrm{~m}$ en partie gauche.

La craie qui forme le substratum des terrains tertiaires de la région parisienne affleure au Sud-Ouest de celle-ci. Elle a été exploitée en souterrain sur environ 20 hectares (dont 8 ha déjà effondrés) à Issy 


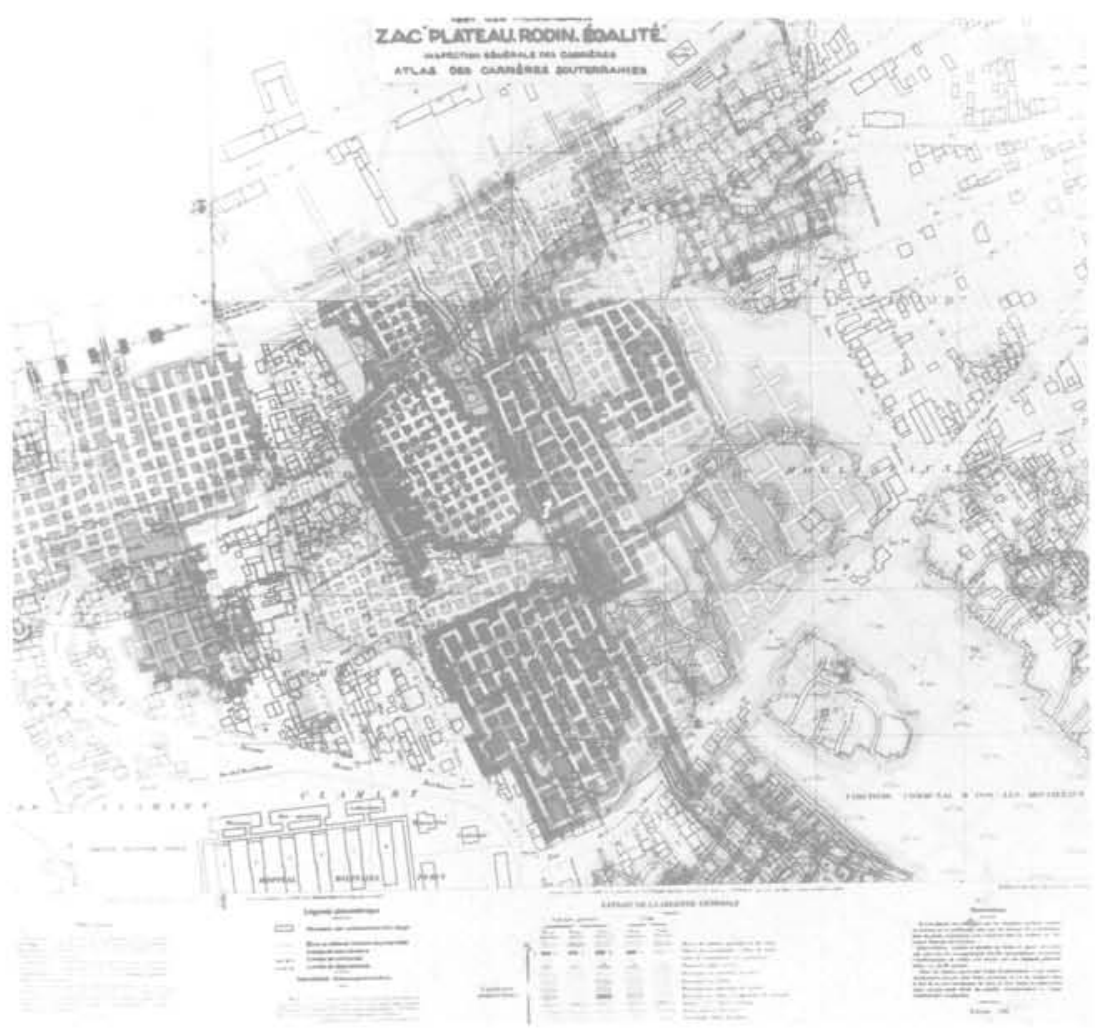

$\triangle$ Fig 1 : La colline d'Issy les Moulineaux

(origine : Inspection Générale des Carrières)

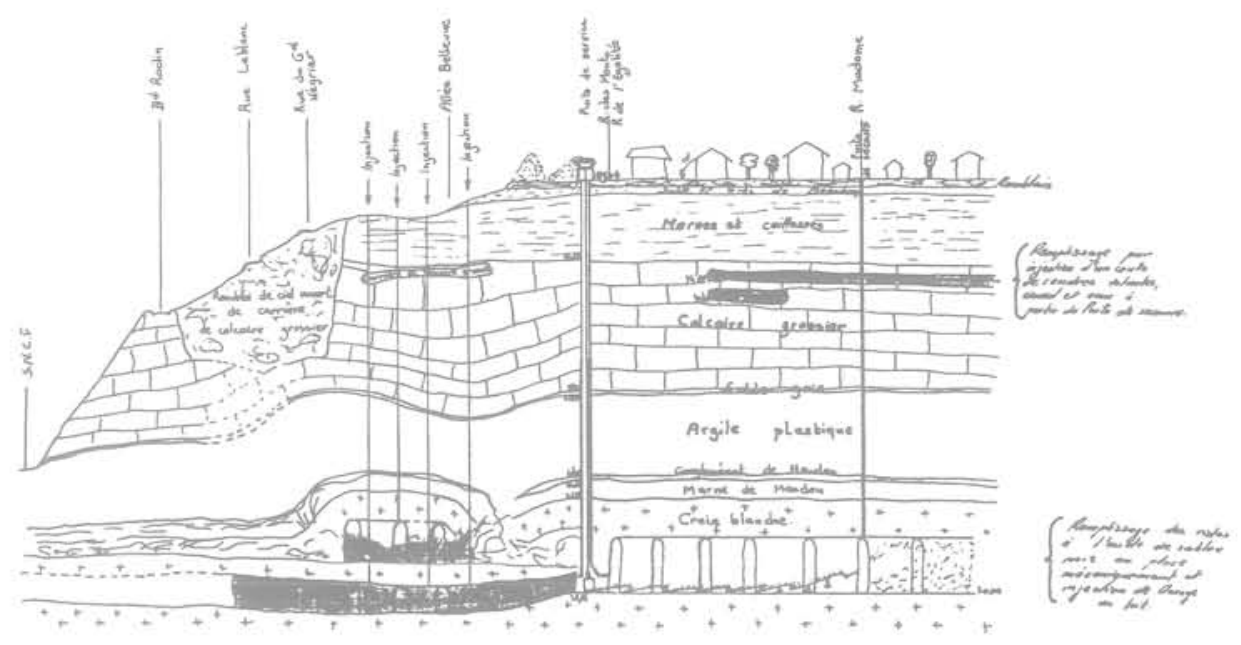

- Coupe et gehenan de prinolpe des travaux 
les Moulineaux et Clamart au 19ème siècle, les dernières exploitations datant de 1925 . Trois effordrements récents, intéressant un ou plusieurs hectares et attribués pour les deux premiers a un écrasement des piliers, ont eu lieu en 1843,1876 et le ler juin 1961 (fig 3 \& 4); du fait de la progression de 1'urbanisation, seul celui de 1961 toucha 1 'habitat ( 21 morts, 36 blessés et destruction d'une vingtaine de bâtiments) (fig 5 \& 6) Dans ce dernier effondrement, on pense que c'est le banc du calcaire grossier qui s'est affaissé brutalement au droit des étaux de masse et après glissement sur l'argile plastique sous-jacente a provoqué l'affaissement des carrières de craie ainsi que des terrains sus-jacents.

Des projets avaient été étudiés en 1957 et 1960 sur des surfaces de 4 à 5 ha par des groupes privés et l'office d'H.L.M. ; ces projets se sont toujours heurtés à l'opposition formelle de l'Inspection des Carrières qui ne concevait pas de ncuvelles constructions sans stabilisation complète du site, opposition confirmée fort justement par le bureau Véritas en décembre 1960, soit donc 6 mois avant l'effondrement.

En fait les carrières étaient régulièrement visitées par l Inspection des Carrières pour essayer au mieux de prévoir une évacuation du site, pensant que l'effondrement serait en 1961 du même type que ceux de 1843 \& 76 . Or 11 semble que les piliers travaillaient à des taux de l'ordre de 20 bars pour une craie dont la résistance à la compression simple est de 22 bars, et que depuis longtemps déjà la masse de calcaire fonctionnait en voute, sa rupture et l'effet de choc a donc largement suffit pour écraser les piliers existants.

Sous l'égide du Cabinet du Premier Ministre, après l'effondrement, la zone a été mise en état de péril et l'Inspection des Carrières a fait procéder à un remplissage de blocage des zones les plus sensibles en vue de protéger les habitations restantes; les travaux n'ont pas été négligeables (prés de 8000000 Francs en 1962) ( fig 7), mais 11 est évident que la collectivité ne pouvait assurer la reprise totale de la colline et s'est donc contentée de la faire évacuer et de mettre en péril tous les bâtiments restants en périphérie de l'effondrement.

\section{3) NATURE DES ETUDES ET HYPOTHESES}

Une première campagne de sondage de la Société S.I.F. avait été réalisée en 1960 pour le compte de 1'O.T.H. et $1^{\prime}$ office d'H.L.M. Cette campagne comportait 6 sondages carottés de 50 à 70 mètres. Une seconde campagne d'une dizaine de sondages carottés fut réalisée par nos soins en 1970 .

C'est de l'ensemble de ces sondages qu'a été déduite la coupe type de la fig 2.

on constate que finalement c'est la couche d'argile plastique qui crée le problème de stabilité ; en effet, c'est hors effondrement la seule masse plastique existante or cette masse n'est plus frettée à l'aval du fait de son affleurement à flanc de coteau. Ses caractéristiques moyennes sont les suivantes :

- teneur en eau 30 \% (inférieure à la limite de platicité)

- densité apparente $20 \mathrm{kN} / \mathrm{m} 3$

- poids spécifique $27 \mathrm{kN} / \mathrm{m} 3$

- indice de plasticité 25 à 60

- résistance à ta compression simple sans contrainte latérale 3 à $510^{5}$ pascal

- densité sèche voisine de $15 \mathrm{kN} / \mathrm{m} 3$

- angle de frottement voisin de $20^{\circ}$

- cohésion de l'ordre de 1.2 à $1.510^{5}$ pascal.

Cette argile est chargée par $30 \mathrm{~m}$ de terrains soit $610^{5}$ pascal.

La craie a pour caractéristiques moyennes :

- densité apparente variant de 15 à $18 \mathrm{kN} / \mathrm{m} 3$

- résistance à la compression simple dans 10 sens perpendiculaire aux couches de craie 20 à $2210^{5}$ pascal.

Mais cette même craie placée dans 1'eau pendant 48 heures devient pateuse sur une épaisseur de 1'ordre de $2 \mathrm{~cm}$ pour des cubes $20 \mathrm{x}$ 20 × 20 et même quelques éléments se détachent et tombent au fond du récipient. Si nous admettons l'existence de voute d'argile au dessus des piliers de craie et une hypothèse de voute sur mur, soit plein ceintre, soit parabolique, avec des fressions intérieures maintenues normales et uniformes, les charges verticales sont de $610^{5}$ pascal, 1 'épaisseur des murs est généralement de 6 mètres et la portée des voutes de 4 mètres, le taux de travail à la naissance est alors de $10^{6}$ pascal ; par contre si au lieu du mur il s'agit de piliers de $6 \times 6 \mathrm{~m}$, le taux de travail à la naissance devient 1.66 $10^{6}$ pascal.

Que peut-on en dédliire sinon que les exploitants avaient un sens très précls des limites qu'il ne fallait pas dépasser.

Or, l'examen de la photo de la fig 8 montre parfaitement ce qui se passe depuis la fin de l'exploitation : les naissances des voutes, sous 1'effet de I'humidité, fonctionnent comme notre échantilion plongé dans l'eau et s'effritent petit à petit, les piliers vont en s'amincissant et la portée des voutes d'une part augmente, mais d'autre part la voute perd ses caractéristiques géométriques parfaites de l'origine (fig 9).

La photo de la fig 10 montre un autre phénomène beaucoup plus génant, car si l'on reprend les fig 3 \& 4 , on constate que les 3 effondrements ont créé un gigantesque barrage à l'écoulement des eaux. Du fait du remplissage des carrières de craie par l'argile plastique, l'eau a monté jusqu'à la zone de rupture de l'argile plastique et s'écoule ensuite par débordement vers la Seine, mais l'ensemble crée non seulement une poussée hydrostatique en pied non négligeable, mais aussi noie les pieds des piliers qui ne se sont pas encore effondrés. 


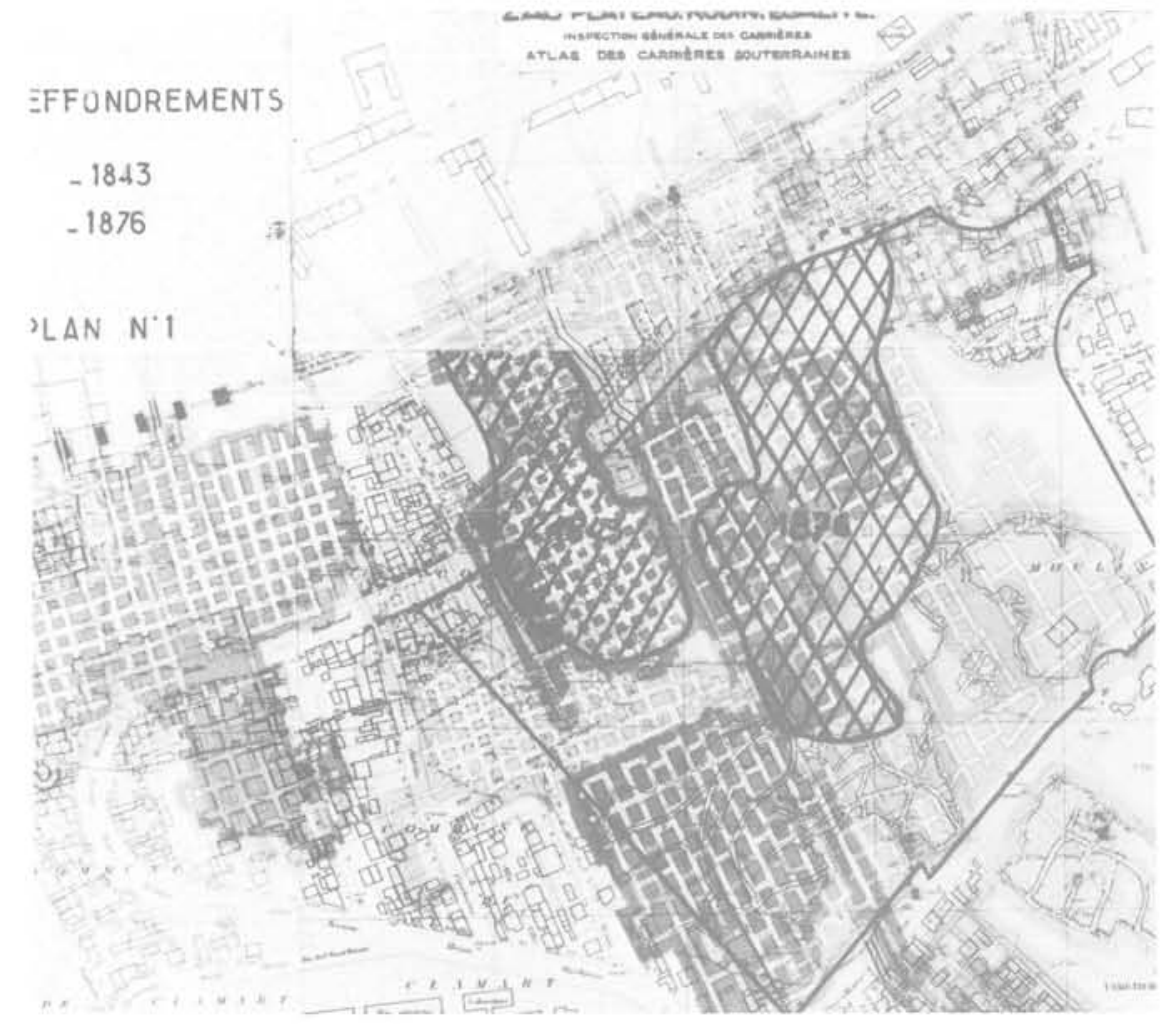

$\Delta$ Fig 3 : Les effondrements de 1845 et 1876

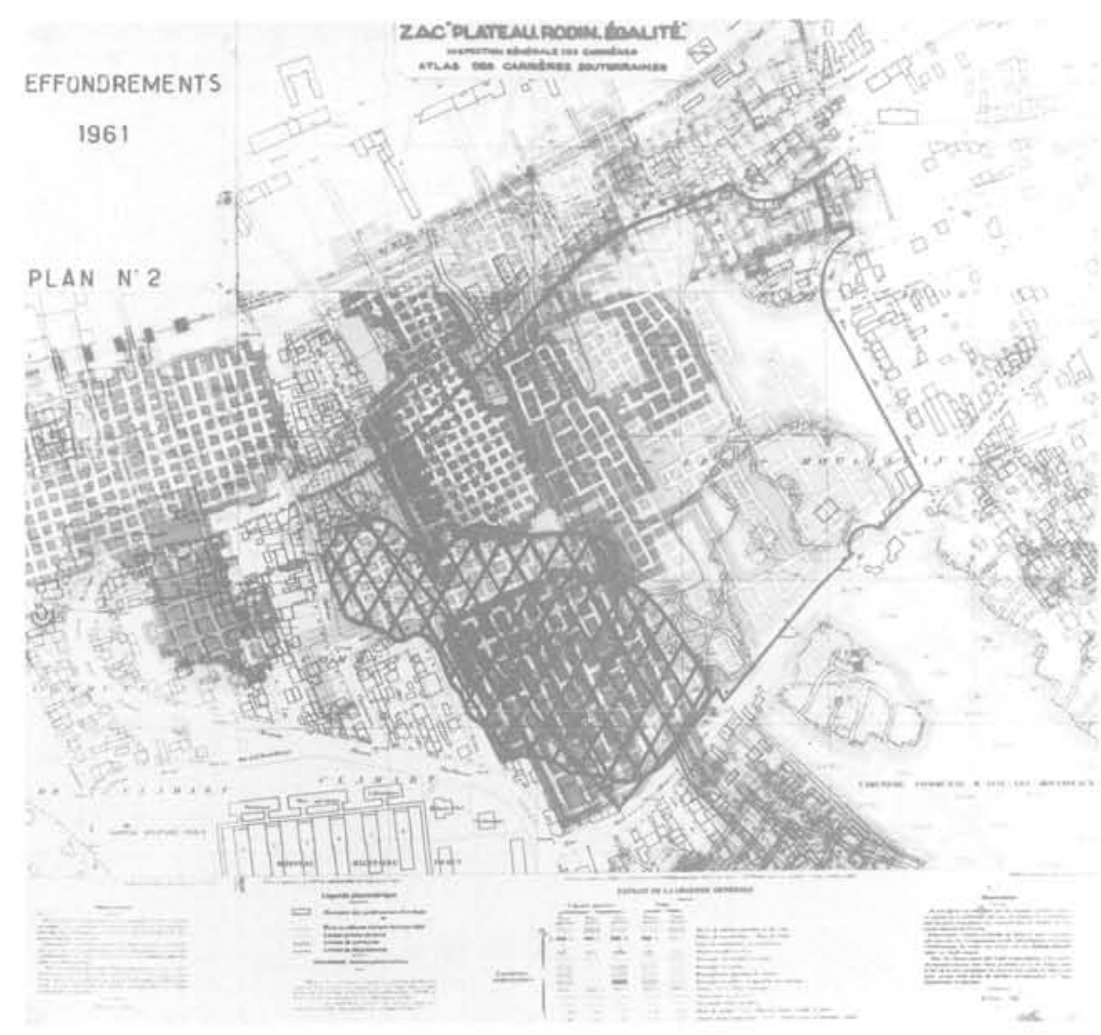

$\Delta$ Fig $4:$ l'effondrement de 1961 

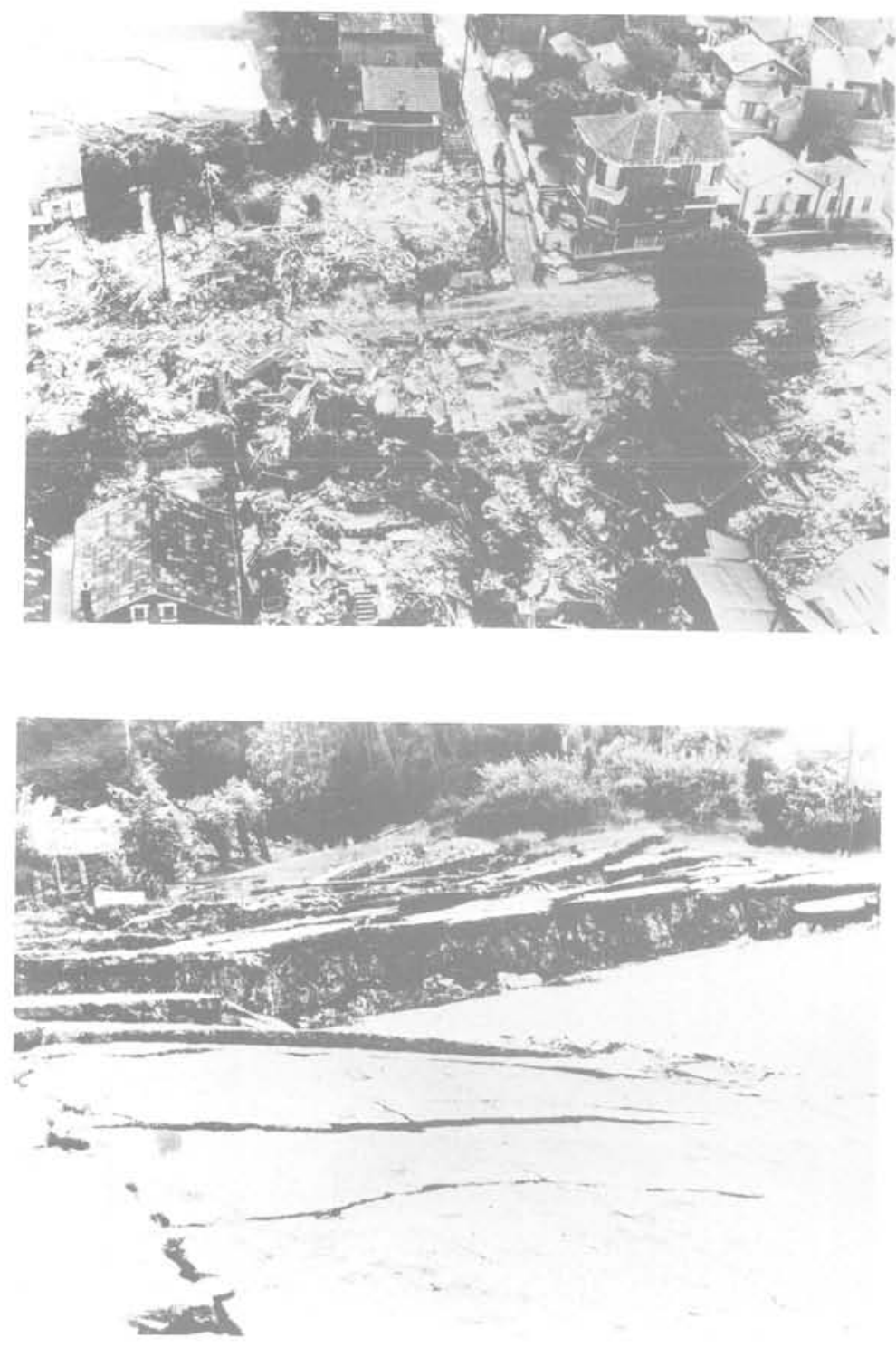

Fig 5 : L'effondrement de 1961 - vue aérienne (origine: Services Techniques Préfecture de Paris)
Fig 6 : L'effondrement de 1961 - affaissements (photo La Hanie)
On peut sommairement conclure que la stabilité est donc réduite par les qualités de I' argile, l'affaiblissement des piliers par altération continue générale et Iocalement par l'eau de la nappe qui après remontée de 7 à $8 \mathrm{~m}$ se situe dans un bassin de rétention de l'ordre de 10 à 15 hectares.

Tout ne pouvant être défavorable, on peut constater sur la fig 1 qu'il existe localement des zones non exploitées sous forme de piliers géants de 1 'ordre de $30 \mathrm{~m} \times 80 \mathrm{~m}$ faisant éperon, et un en particulier trapézoldale très net sur les fig 3 \& 4 qui a arrêté les 3 effondrements. A l'échelle de galerie de $4 \mathrm{~m}$ de large, ce pilier a environ $30 \mathrm{~m} \times 30 \mathrm{~m}$.

De plus, du fait de l'anticlinale de Meudon, l'argile plastique sublt d'une manière assez générale un basculement vers 1 'amont.

\section{4) CONCEPTION DU PROJET :}

Il a été décidé d'admettre les hypothèses suivantes :

Si dans la zone non effondrée on reconstitue la masse de craie par un matériau ayant des caractéristiques à l'écrasement de 20 à 25 bars, ont peut reconstituer la totalité de lá colline dans ces zones, l'accès étant possible à des engins de nettoyage des remblais et déchets pouvant exister

Pour les effondrements de 1843 \& 1876 qui sont à flanc de coteaux, le terrain peut être traité par injection sur une épaisseur de 55 mètres depuis le niveau bas des carrières jusqu'au niveau du sol ; ceci devrait permettre d'éviter des mouvements en grande masse mais pas les mouvements localisés et les tassements dûs au fluage de l'argile vers la vallée ; ces zones ne peuvent donc être utilisées que comme espaces verts. 


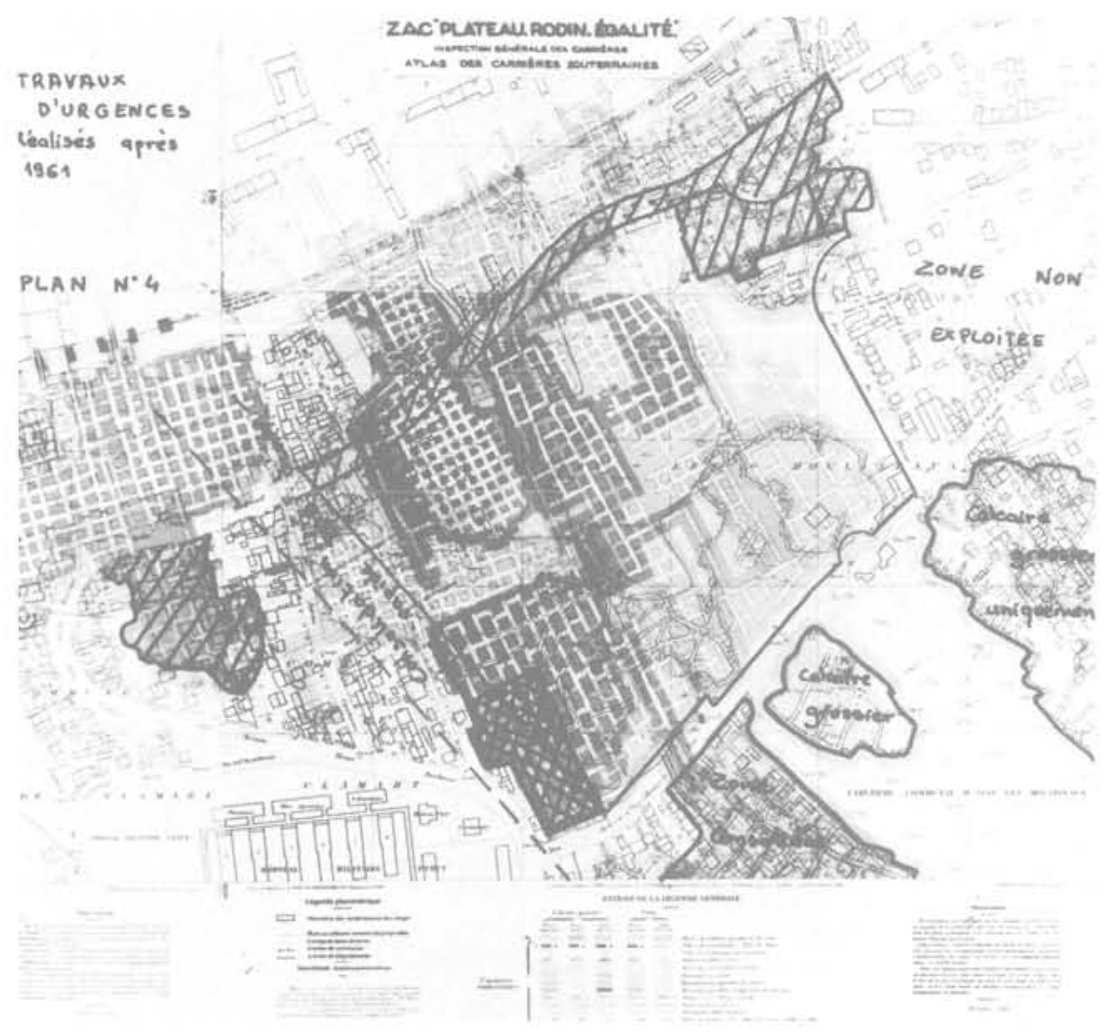

$\Delta$ Fig 7 : Les travaux d'urgence après I'effondrement

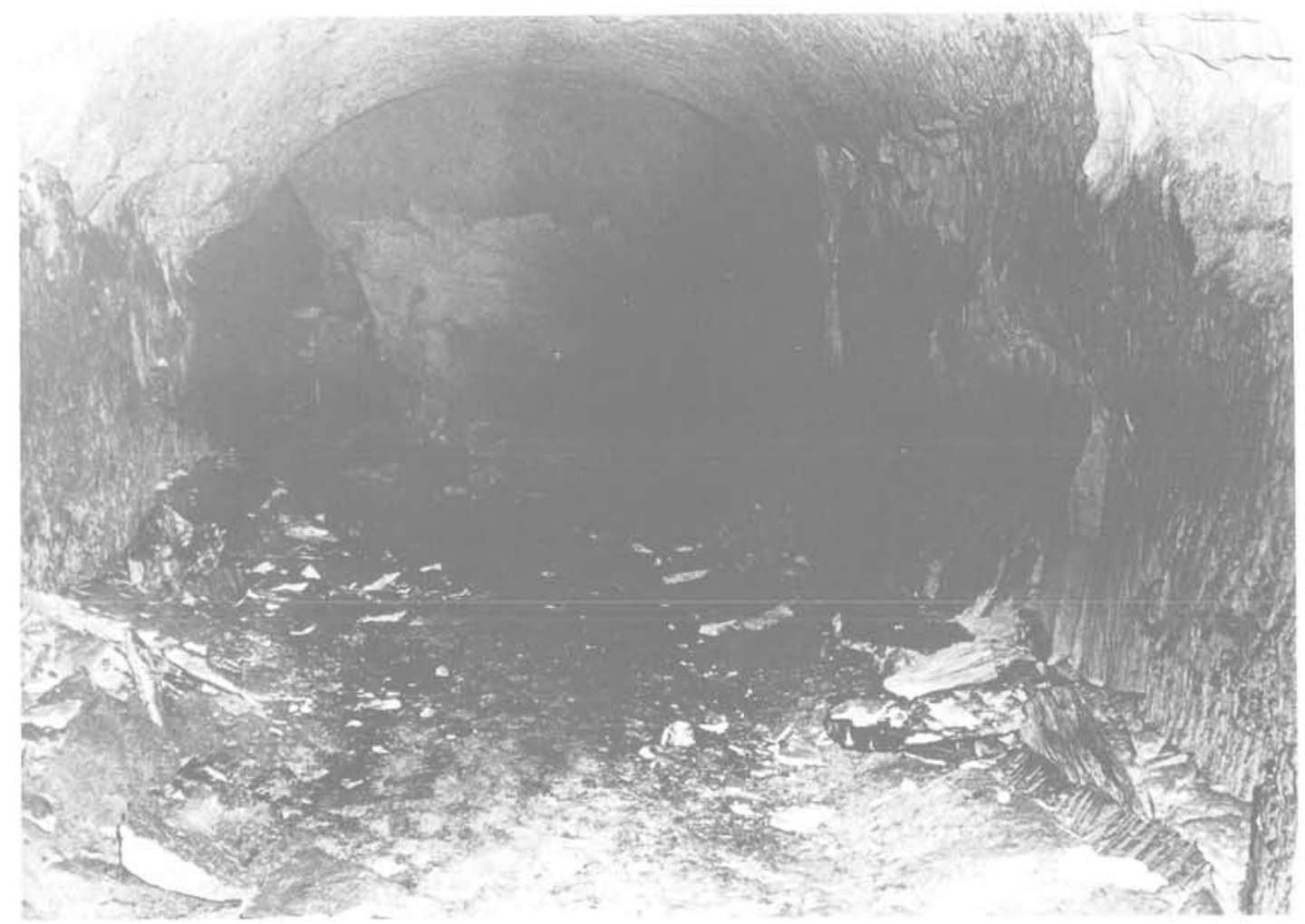

$\triangle$ Fig 8 : L'altération des piliers

(photo CESSDEMOND) 


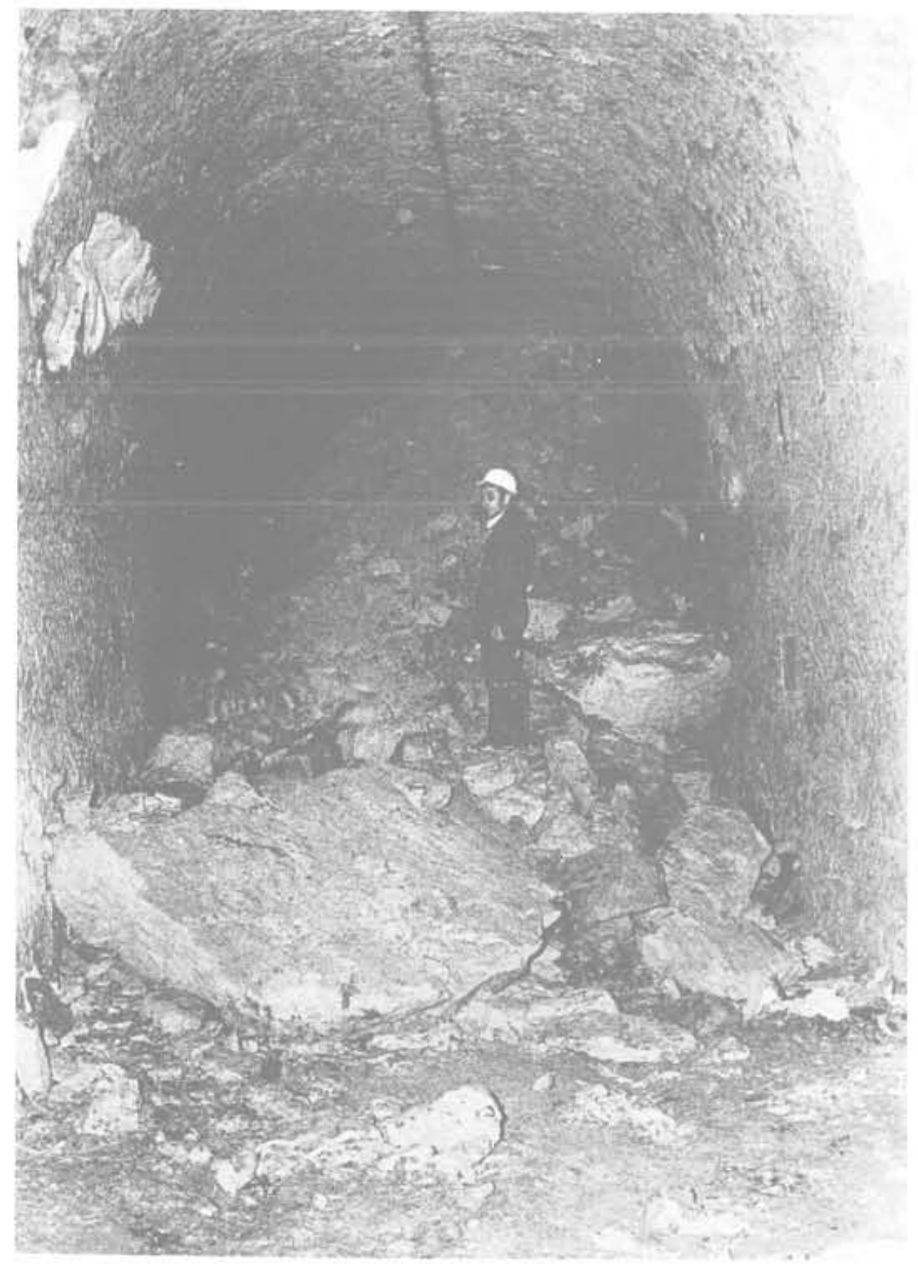

Fig 9: Galerie en état en limite d'effondrement (photo CESSDEMOND)

L'effondrement de 1961 se situant sur le plateau, peut être traité par injection de bas en haut et pourra être construit sous réserve de n'apporter aucune charge, c'est à dire đe réaliser des bâtiments fondés sur pieux de $55 \mathrm{~m}$, de gros diamètre, armés et chemisés contre le frottement négatif.

Ces utilisations sont associées à deux obligations techniques : ne pas charger le terrain d'une part, d'autre part assurer l'écoulement de l'eau.

Les bâtiments non fondés sur pleux représentent environ 240000 tonnes ; il s'agira donc de décharger la tête de colline de ce poids, ce qui sera réalisé par une fouille sous les bâtiments de $12000 \mathrm{~m} 2$ sur $16 \mathrm{~m}$ de profondeur soit environ 345000 tonnes, ce qui est relativement alsé du fait d'un besoin très important de parking dans cette partie de la ville En ce qui concerne l'écoulement de l'eau, une galerie perpendiculaire à la pente est lancée entre les effondrements de 1843 \& 1876 d'une part et 1961 \& 1876 d'autre part ; cette galerie drainante visitable par un puits en amont équipé de $60 \mathrm{~m}$ de profondeur et $1.60 \mathrm{~m}$ de diamètre aboutit à une fosse de rétention permettant d'infiltrer l'eau dans une faille

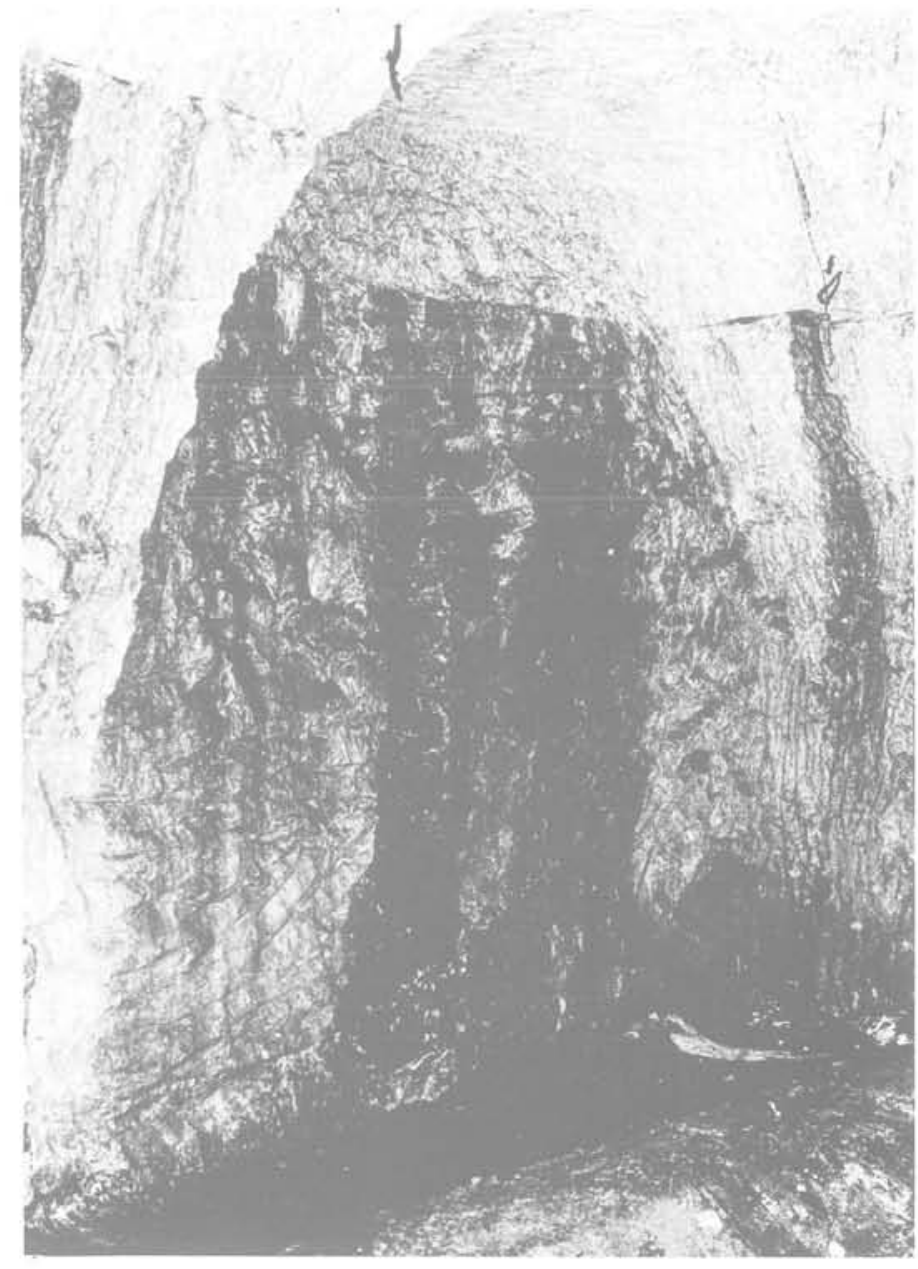

Fig 10 : Remontée de la nappe après I'effondrement (photo CESSDEMOND)

de la craie qui a été découverte par l'inspection générale des carrières, mais un système de relevage par pompe permet de parer ultérieurement au risque de colmatage.

Sur cette galerie arrivent des drains subhorizontaux variant de 100 à $300 \mathrm{ml}$ de long . et permettant d'aller "chercher" l'eau derrière les effondrements.

Pour la zone à gauche de la future voie rapide (fig 7) où les carrières sont actuellement utilisées par des marchands de vins ou légumes, la consolidation consistera en une association de remplissage traditionnel et de voutes en béton armé de consolidation.

\section{5) EXECUTION DES TRAVAUX :}

L'ensemble des travaux, sauf les puits, a été réalisé à ce jour par la Société Auxiliaire d'Entreprises associée à la Société La Souterraine. Le remplissage s'est réalisé à l'aide de 800 sondages tubés après nettoyage semi-mécanique des carrières accessibles. Les photos des fig 11 \& 12 montrent parfaitement les coulis mis en oeuvre dans les zones accessibles. Le traitement par injection est trop classique pour qu'il y ait un intérêt quelconque à I'évoquer ici. 
Dans les zones non accessibles, les contrôles ont été réalisés de façon très classique par essais Lugeon, car le programme de contrôle prévu par caméra de télévision descendue dans des sondages de $250 \mathrm{~mm}$ a été un parfait échec, la distinction entre la craie et le coulis sablon-ciment étant très difficile à réaliser même en photos couleurs.

En ce qui concerne la stabilité, il a été mis en oeuvre deux réseaux de sondages de contrôle :

- le premier équipé d'inclinomètres qui n' ont rien constaté de particulier sauf l'effet classique d'écartement par soulèvement du fond de fouille d'un terrassement de $12000 \mathrm{~m} 2$ sur $16 \mathrm{~m}$ de hauteur, le chargement apporté par les bâtiments $(R+30+6$ sous-sols) ayant à peu près rétabli le basculement.

- le second équipé de tassomètres mis en oeuvre dans les 3 zones d'effondrement pour essayer de déterminer I'évolution du fluage. L'interprétation des résultats ne nous semble pas très convaincante dans l'état actuel des mesures (les travaux d' injection sont terminés depuis 4 ans) ; il. y a certainement des mouvements qui peuvent être aussi bien dûs à des phénomènes de retrait des coulis qu'à des phénomènes de tassement dûs au mouvement des terres nécessaires à l'aménagement de l'espace vert de $30000 \mathrm{~m} 2$ actuellement en cours.

On peut dire de toute façon, qu'actuellement, les points les plus sensibles de la colline sont colmatés, mais la colline ne sera pas définitivement stabilisée avant 1985.

Le programme de fondation restant à réaliser étant de l'ordre de 50000000 Frs, valeur actuelle, on peut dire qu'il ne semble plus y avoir de risques d'effondrement de très grande taille sur la colline elle-même, la seule partie restant actuellement sensible étant le flanc de coteau.

\section{6) CONCLUSIONS :}

A notre époque où le problème des carrières et des vides de dissolution est soulevé dans tous les coins de France, il semble intéressant de revenir à des idées saines de statique générale des sites, et abandonner les solutions de remplissage ou de traitement aléatoires. Il est certain que les coûts financiers sont très importants et pour la colline d'Issy les Mx, entre les travaux de l'Etat, de la Municipalité, et du Privé, le coût final devrait dépasser les 100000000 Frs actuels, mais nous estimons que cet argent est dépensé pour une certitude de stabilité.

Nous avons évité d'évoquer les études faites par notre laboratoire sur les stabilités des cendres, l'utilisation des sables de fonderie les coulis à composants chimiques qui ont pu nous être présentés pour résoudre le problème d'amener $300000 \mathrm{~m} 3$ de matériaux sur le site. L'économie est symbolique par rapport au problème posé.

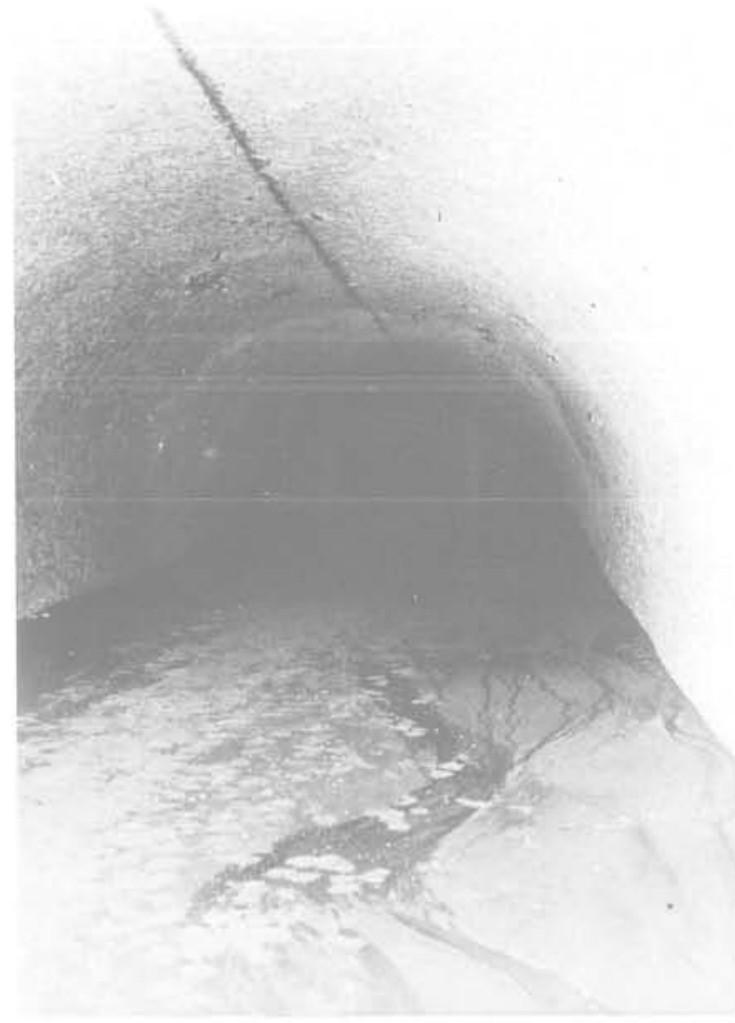

Fig 11 : Le coulis en cours de mise en oeuvre dans une galerie (photo SAE)

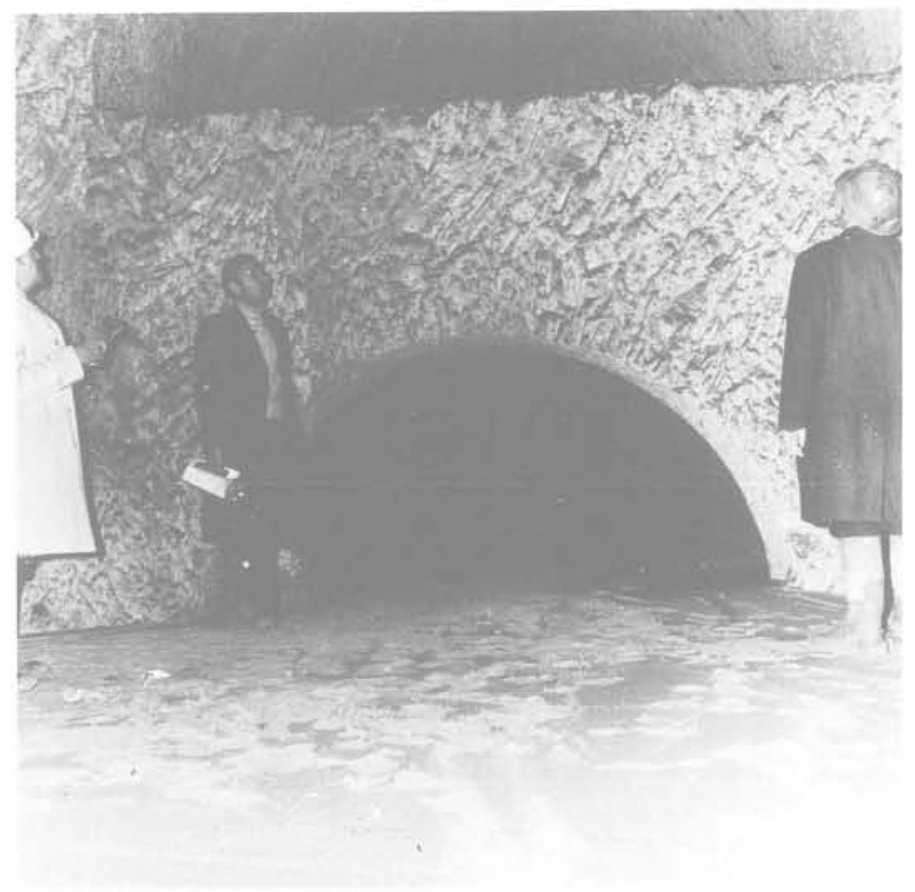

Fig 12 : Le coulis après 24 heures visite de contrôle (photo SAE) 